\title{
BiologicalNetworks - tools enabling the integration of multi-scale data for the host-pathogen studies
}

Sergey Kozhenkov', Mayya Sedova', Yulia Dubinina ${ }^{1}$, Amarnath Gupta', Animesh Ray ${ }^{3}$, Julia Ponomarenko ${ }^{1,2}$, Michael Baitaluk ${ }^{1 *}$

\begin{abstract}
Background: Understanding of immune response mechanisms of pathogen-infected host requires multi-scale analysis of genome-wide data. Data integration methods have proved useful to the study of biological processes in model organisms, but their systematic application to the study of host immune system response to a pathogen and human disease is still in the initial stage.

Results: To study host-pathogen interaction on the systems biology level, an extension to the previously described BiologicalNetworks system is proposed. The developed methods and data integration and querying tools allow simplifying and streamlining the process of integration of diverse experimental data types, including molecular interactions and phylogenetic classifications, genomic sequences and protein structure information, gene expression and virulence data for pathogen-related studies. The data can be integrated from the databases and user's files for both public and private use.

Conclusions: The developed system can be used for the systems-level analysis of host-pathogen interactions, including host molecular pathways that are induced/repressed during the infections, co-expressed genes, and conserved transcription factor binding sites. Previously unknown to be associated with the influenza infection genes were identified and suggested for further investigation as potential drug targets. Developed methods and data are available through the Java application (from BiologicalNetworks program at http://www.

biologicalnetworks.org) and web interface (at http://flu.sdsc.edu).
\end{abstract}

\section{Background}

Public health initiatives increasingly recognize the importance of the cross-scale data integration, such as mounting a data-driven risk assessment of potential pandemic outbreak in specific geographical locations or discovering novel therapeutic approaches [1-6]. For example, to facilitate the study of the Influenza infection outbreaks $[7,8]$, it is desirable to apply the systems biology approach that requires integration of heterogeneous data from various domains of knowledge: flight paths of migrating birds, animals and humans; virological aspects, such as the efficiency with which the virus can be transmitted from the infected subject; cellular

\footnotetext{
* Correspondence: baitaluk@sdsc.edu

${ }^{1}$ San Diego Supercomputer Center, University of California San Diego, 9500 Gilman Drive, La Jolla, CA, 92093, USA

Full list of author information is available at the end of the article
}

phenomena, such as interaction of viral proteins with surface receptors in the inner and outer respiratory tracts of hosts; phylogenetic properties of viral strains and viral proteins; structural properties of proteins; and molecular interactions of host and virus proteins to each other and small molecules [9-11]. Thus, there is a need in the integration system able to integrate heterogeneous biological and clinical data and enable crossdomain and cross-scale analyses of those data.

Experimental data on host-pathogen interaction are distributed throughout many heterogeneous data sources. Among the integration systems enabling studying host-pathogen interactions at multi-level scale are PHI-base [12], PHIDIAS [13], PIG [14], IVDB (Influenza Virus Database) [15], and the NCBI Influenza Virus Database [16]. In these resources, data sources are integrated mostly through URL links. Despite the active

\section{Biomed Central}


research in the field, most of the published data concerning host-pathogen interactions [17-28] are not available for the study in the concert with other data: they can be accessed only as supplemental tables to the papers and at best visualized using the network visualization and navigation tools, such as Cytoscape [29], GenMAPP [30], GeneSpring (Agilent). These solutions, however, do not allow integration of orthogonal types of data, such as 3D protein structures or sequences of gene regulatory regions, for example. They also do not allow phylogenetic, orthologous or phylogeographic analysis that is necessary, considering the fact that the detail experimental analysis of host-pathogen interactions for each of the existing, emerging and reemerging pathogens is not feasible.

At the same time, existing link-based integration systems, such as Entrez [31], Ensembl [32], or BioMart [33], provide limited capabilities for analysis of hostpathogen interactions and pathways specifically. While most heterogeneous data integration systems, or warehouses, are either domain-specific-for example, STRING [34], GeneCards [35], or PharmGKB [36] deal with genomic data exclusively-or do not allow sequence search and annotation, for example, ONDEX [37], BIOZON [38], or BNDB [39].

In this paper, the approach at cross-scale data integration to study host-pathogen interactions is proposed and demonstrated on a study of the Influenza infection. The proposed system is an extension of the previously developed BiologicalNetworks [40,41] and IntegromeDB [42]. It represents a general-purpose graph warehouse with its own data definition and query language, augmented with data types for biological entities. Developed methods and implemented solutions for the integration, search, visualization and analysis of host-pathogen interaction data are available through the BiologicalNetworks application http://www.biologicalnetworks.org and web interface http://flu.sdsc.edu; Demo page: http://flu.sdsc. edu/examples.jsp.

\section{Methods}

\section{System}

The architecture of the system, data integration and mapping procedures, database schema, ontology model and data query engine are described in detail elsewhere [42]. Therefore, only brief description is provided here. Data integration and mapping to the internal database is fully automated and based on Semantic Web technologies and Web Ontology Language (OWL) http://www. w3.org/TR/owl-ref. The IntegromeDB [42] internal database schema is RDF-compatible (Resource Description Framework; http://www.w3.org/RDF/); that is, it stores biological data in an RDF-compatible format, the standard format of the Semantic Web [43]. The database architecture and database schema are provided at http:// www.BiologicalNetworks.net/Database/tut0.php. The ontology is available as an OWL file at http://flu.sdsc. edu/bionetsonto.jsp.

\section{Data}

The full list of integrated databases and statistics are provided at http://www.biologicalnetworks.net/Database/ tut5.php [42]. To enable research on host-pathogen interactions, in addition to previous integrated data on genome and protein sequences, gene expression and regulation data, protein-protein and protein-DNA interactions [42], the following data were integrated (Table 1):

- Phylogenetic trees that connect host and pathogen proteins/genes with orthologs/homologs in model organisms (with their molecular sequence, structure, expression and interaction data). These data were obtained from PhyloFacts database [44].

- Literature-curated information on physiological effects of pathogen infection in experimental systems (including cell cultures and in vivo models)

- Virulence data about mortality/morbidity information related to isolate and incidence, isolated organism's sequence data (from WHO statistics)

- Epidemiological data on infection occurrence, pathogen culture sites and dates, migration data of vectors, past movement data of infected individuals, etc. (from NCBI Influenza Virus Resource).

Additional experimental data sets on host-pathogen interactions integrated into our system include human interactomes used by Influenza virus, HIV, HCV, dengue virus and West Nile virus (WNV) (based on the results reported in [17,22-28]).

\section{Data access}

The web page http://flu.sdsc.edu (Figure 1) provides genomes/pathogenic strains searches by keywords and genomic and protein sequences, statistics on integrated data by category and data source, information relating to retrieved properties by data sources for each gene/ protein that can be accessed from the query result page, and data inconsistencies in public data. The web site was designed primarily for the purpose of giving the user an opportunity to quickly search for phylogenetic relations among sequence strains and perform at integrated data rather than to provide complex data analysis capabilities, which are implemented in the BiologicalNetworks application, which can be downloaded at http://www.BiologicalNetworks.org (Figure 1).

To integrate the user's data into the system, the web page http://flu.sdsc.edu/integration.jsp (see Section 'Integration of user's data' below) is provided. The data will 
Table 1 Integrated data for the Host-Pathogen interaction studies

\begin{tabular}{llll}
\hline & Host & Pathogen & Host-Pathogen \\
\hline Genome strains & - & 22949 & $11843(\mathrm{H})$ \\
\hline Genome Sequences (complete genomes) & 354 & 3994 & - \\
\hline Protein Sequences & 99117 & 58218 & - \\
\hline cDNA library Sequences (+conditions, tissue sources) & 79431 & 47175 & - \\
\hline Gene expression & 12983 & - & 29 \\
\hline 3D structures & 135819 & 380 & - \\
\hline Protein- protein interactions/Reactions/Relations & $>200$ 000 000 in more than 1000 organisms & 650 & 250 \\
\hline Phylogeny & 9 phylogenetic tree libraries from PhyloFacts database $[44]$ & \\
\hline Virulence & Statistics on confirmed Human cases of Influenza reported to WHO \\
\hline Epidemiology & Infection occurrence, pathogen culture sites, dates, migration data vectors, movement \\
\hline
\end{tabular}

become public, but unless curated by the data administrators, will remain "tagged" as 'uncurated' under the contributor's name. The user's data integration procedure consists of 3 simple steps: 1) User registration, 2) Data Mapping and 3) Data integration (see Section 'Integration of user's data' below). To be integrated, the data needs to be in the table format.

\section{Internal Data Model and Data Structures}

The internal schema of the BiologicalNetworks database is shown in Figure 2. Four orthogonal types of biological data-graphs, sequences, histograms, and tree structures-are integrated to enable multi-scale data analysis for the host-pathogen studies. In the process of integration, all external data types (Figure 2A) are transformed into graph and tree data structures (Figure 2B): onedimensional sequence data (e.g. sequences) into interval trees; two- and three-dimensional data (e.g. images) into $\mathrm{R}$-trees. To keep the number of the index structures small, a single interval tree is created per chromosome instead of per annotated DNA sequence regions, and the images of the same resolution are referenced with

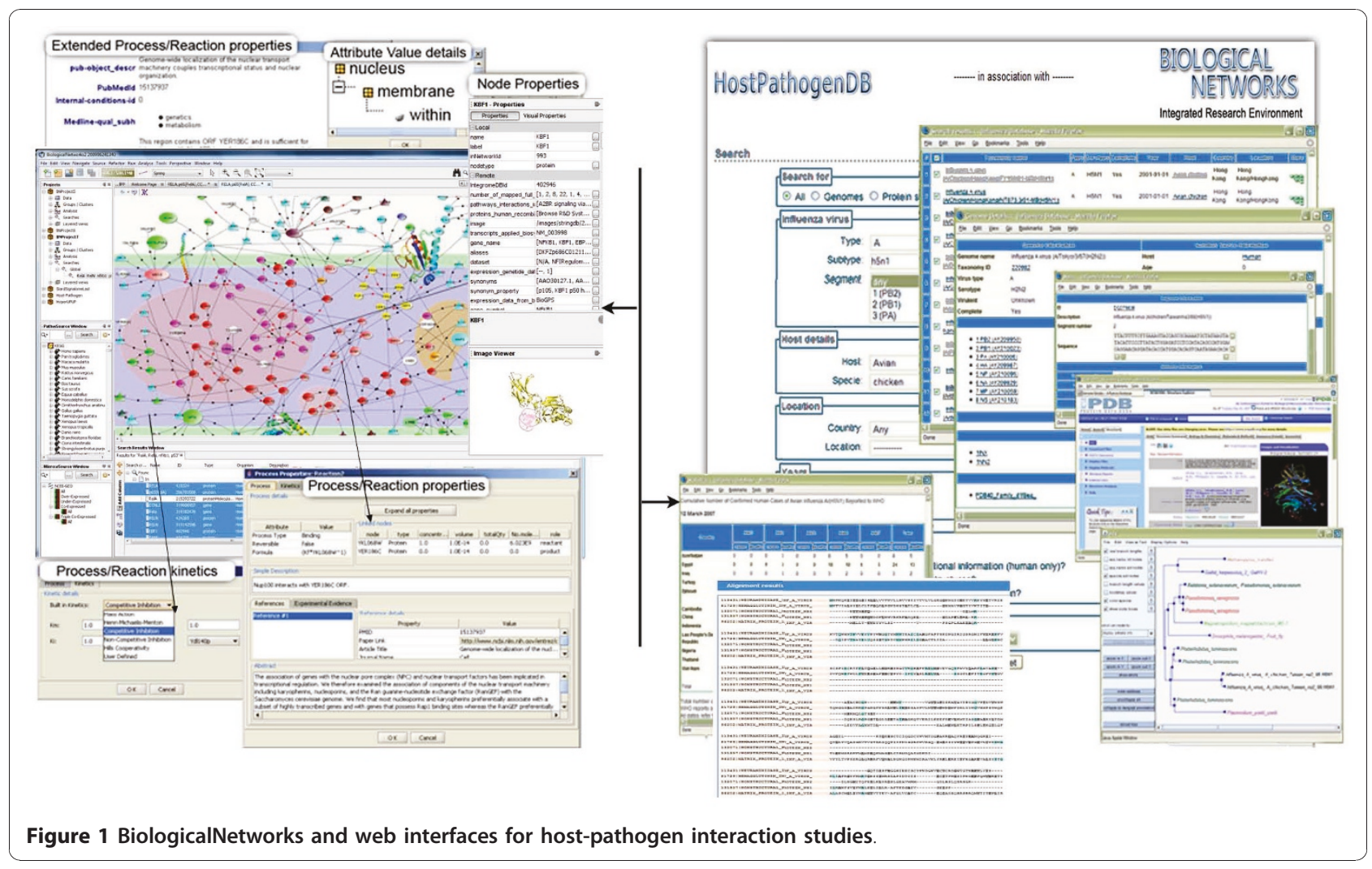




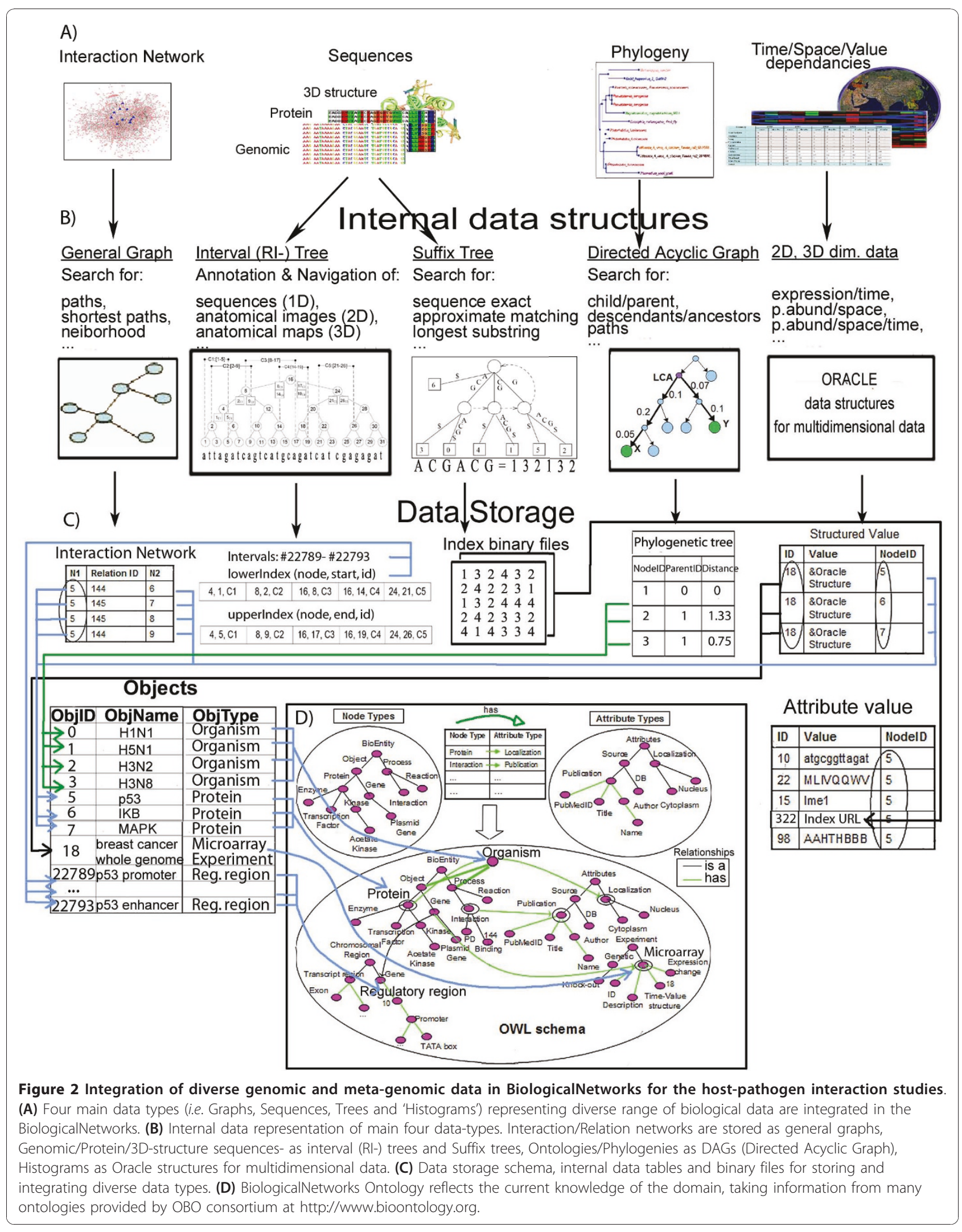


respect to the same coordinate system and placed in a single R-tree. The examples of operations on RI-trees that are used in BiologicalNetworks for Navigation and Annotation of multiply overlapping gene regulatory regions, protein binding regions, disease and geographical maps are provided in the Additional File 1, Section S1.1.

Nodes of the interval and R-trees (Figure 2C) are connected to ontology nodes (Figure 2D) through the internal Objects and Attribute values tables that are in turn connected through BioNets Ontology http://flu.sdsc.edu/ bionetsonto.jsp (Figure 2D). BioNets ontology consists of three parts: (i) the general-purpose Basic Ontology, which is the modification of BioPAX Level 2 ontology; (ii) manually (by the authors) mapped to the basic ontology 25 selected OBO ontologies (specified at http://flu.sdsc.edu/bionetsonto.jsp and provided in the Additional File 1, Figure S2); and (iii) 72 OBO ontologies that are mapped to each other and the selected 25 ontologies as provided by the OBO consortium. The basic ontology and mappings to the selected 25 ontologies are provided in the file basic.owl available at http:// flu.sdsc.edu/bionetsonto.jsp under "MappinSuperClass" class and "sameAs" properties.

A new ontology can be introduced without modification of the BioNets ontology classes and through 'ontology mapping' [43]. For example, for a SequenceOntology that maps a class Gene 'SO:12345', a new class 'mappingSO:012345' will be generated using 'same_as' relation. More detailed information on BioNets ontology is provided in the Additional File 1, Section S1.2.

Versatile suffix tree structures (Figure 2B) are used to solve a variety of sequence-based problems, such as exact and approximate matching, database querying, and finding the longest common substrings [45]. A variety of efficient in-memory suffix tree construction algorithms have been proposed [45-50], that are scalable with extremely large (for example, the human and mouse genomes are approximately $3 \mathrm{Gbp}$ and $2.5 \mathrm{Gbp}$ long, respectively) sequences. External biological sequences are transformed to internal suffix tree structures, using TRELLIS algorithm [51]; sequence search operations such as exact sequence search, best match, and longest substring are allowed. Suffix tree representation of genomic/protein sequences is stored as indexed binary files and is mapped to the database sequence objects as property values (Figure 2B and 2C (right)).

\section{Data Querying}

As different categories of data are added to the system, it becomes critical to have an augmented (internal) query language that provides constructs (operators and functions) to search, manipulate and query the data. The developed for this purpose the BioNetQL query language is used behind the user interface in the
BiologicalNetwork application. It can be also used by the users accessing the database directly through the API; for example, our database is extensively used through the BioNetQL API in the CAMERA metagenomic project [52]. The syntax of BioNetQL and its distinction from SQL and SPARQL are considered in the Additional File 1, Section S1.3.

To enable systems-level study of host-pathogen interaction, in addition to the query capabilities described previously [42], a number of new methods were implemented that now allow answering the specific questions concerning host-pathogen interactions. For example, the following questions: (1) What is the evolutionary distance between two specified genome sequences? (2) Which genome sequences are within the specified evolutionary distance from a given genome? (3) What is the probability of a given protein/gene sequence to be virulent? and (4) What is the probability of a protein of a given 3D structure to be virulent? Evolutionary distance was chosen to rationalize the information integration scheme of our database, because virus properties, such as virulence, infectivity, host-specificity, geographic locations, morbidity in an epidemic, or host-specific reactions are related by evolutionary lineages [53].

To address the first question, the database can be searched for evolutionary distances between two specified genome sequences of different species/strains/isolates, executing the following three queries (Figure 3A, $B)$ : (1) reconstruct the PhyloTree containing both sequences; (2) find the least common ancestor (LCA) for two sequences; and (3) find the sum of distances from each of the two sequences to the LCA.

To find the answer to the second question-Which genome sequences are within the evolutionary distance $\mathrm{L}_{\max }$ from a given genome $\mathrm{X}$ ?-the following queries

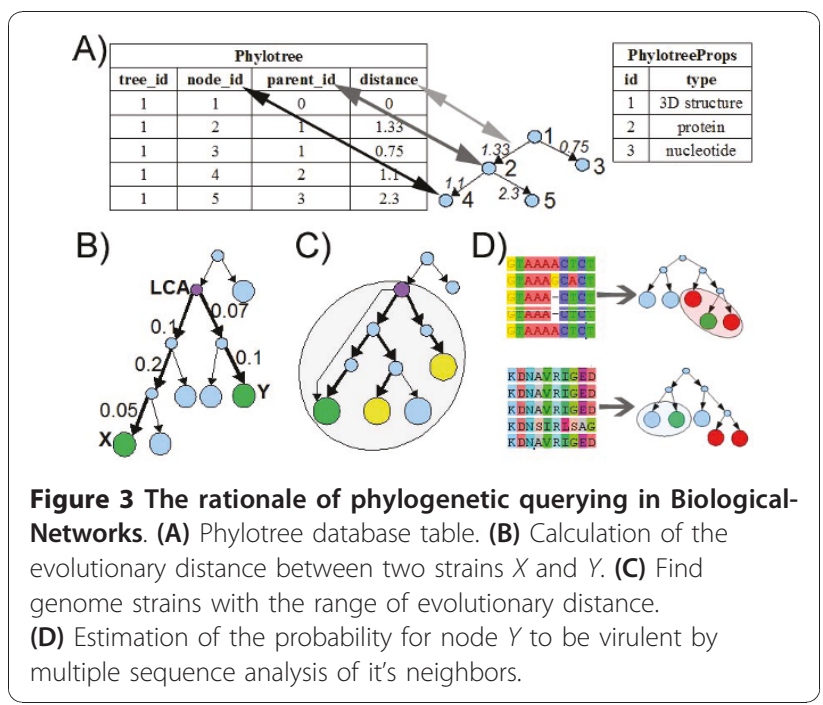


need to be executed (Figure 3C): (1) reconstruct the phylogenetic tree containing $\mathrm{X}$; (2) find the ancestor A of the node $\mathrm{X}$ that is no farther than $\mathrm{L}_{\max }$ from $\mathrm{X}$; and (3) in the ancestor A rooted sub-tree, find the nodes at the distance $\mathrm{L}_{\max }$ from $\mathrm{X}$.

To predict the virulence of a species/strain/isolate by its gene/protein sequence, the published method for virulence evaluation of low and high pathogenic avian influenza LPAI and HPAI [53] was implemented in the system. Using this method, it was shown that HPAI strains in addition to phylogenetic grouping exhibit grouping by geographical region [53]. The following sequence of queries allows applying the method (Figure 3D): (1) recalculate/modify the phylogenetic tree based on multiple sequence alignment; (2) find the nodes nearest to a given node X; and (3) calculate the probability for the node $\mathrm{X}$ to be virulent if its neighbors are known to be virulent or not virulent.

Similarly, the probability of a protein of a given 3D structure to be virulent can be estimated, using the phylogenetic tree built based on 3D structure multiple sequence alignment (Figure 3D).

To enable the aforementioned queries, a number of bioinformatics methods were implemented in BiologicalNetworks both in house and by the others to: reconstruct the phylogenetic tree, recalculate/modify the phylogenetic tree, for multiple sequence alignment $[54,55]$.

Also, for identifying phylogenetically conserved transcription factor binding sites in the gene regulatory regions, the method [56] was applied to the promoter sequences ( the region from -500 to +500 bp relative to the transcription start site) of all integrated genes, using known binding sites that have been integrated in the IntegromeDB [42]. Identified gene pairs and their conserved binding sites were integrated into the system. In addition to the queries by keywords, this data can be queried by sequences (the examples are given in the Application section). The implemented in BiologicalNetworks approach to the sequence querying is discussed in detail elsewhere [42].

\section{Results and Discussion}

The severity of flu pandemic outbreaks, including the recent one of the swine-origin H1N1 influenza virus, and widespread resistance to the existing antiviral drugs demand for new therapeutics targeting host factors. Identification of host genes involved in the virushost interactions is the first step towards developing such therapeutics [17]. In this work, we attempted to identify these genes, analyzing the broad spectrum of publicly available data on influenza viruses and infections, using the BiologicalNetworks application (Figure 4A-D).
To find the potential therapeutic targets in the host, we first identified the genes that were differentially expressed in mouse and human in response to the influenza infection and interacted with the virus and to each other. Then, using the constructed interaction network, we studied the proteins that directly interacted with the virus and co-expressed genes. Sub-networks that were induced/repressed at different stages of the influenza infection were also analyzed. In the result of analysis of co-expressed genes and transcription factor binding sites in their promoters, 118 genes were identified as potential candidates for further investigation; after filtering the genes that are known to be associated with influenza infections, 7 genes were obtained.

Using the influenza viruses as an example, in the following four sections we demonstrate and discuss different types of host-pathogen interactions that are available in BiologicalNetworks. Since at the moment of the analysis, the data were available for only two influenza proteins, neuraminidase and hemagglutinin, the provided below analysis was narrowed down to these two viral proteins. All discussed queries are provided in detail in the Additional file 1, Section S1.4.

The last two sections show additional capabilities of the system, phylogeographical analysis and integration of the user's data, following by the comparison with other systems designed for studying host-pathogen interactions.

\section{Building the influenza virus-host interaction network}

To construct the interaction network for further study, BiologicalNetworks was first queried for the host proteins interacting with the virus and localized in nucleus or cytoplasm (Query 1, Additional File 1, Section S1.4). This query returns the set of pair-wise interactions in the form of a graph. Then, BiologicalNetworks was searched for the genes that were differentially expressed and related to the influenza infection in the microarray experiments (Query 2 Additional File 1, Section S1.4). Since different experiments have different number of time points and conditions, data from every experiment were normalized (for details see Additional File 1, Section S1.4).

Finally, for the discovered genes, we extracted all known interactions among host proteins. Thus, among 3,972 differentially expressed genes in mouse, $~ 12,000$ interactions were found for the proteins implicated in the influenza expression data set. After narrowing the search to the curated interactions from HPRD and BIND databases, we obtained the resulting network consisting of 4,592 interactions among 1,950 molecules (Figure 4E). Due to limited data available in human, the network for human was much smaller and contained only 413 influenza-human and human-human interactions. Now, the constructed networks can be examined in detail. 


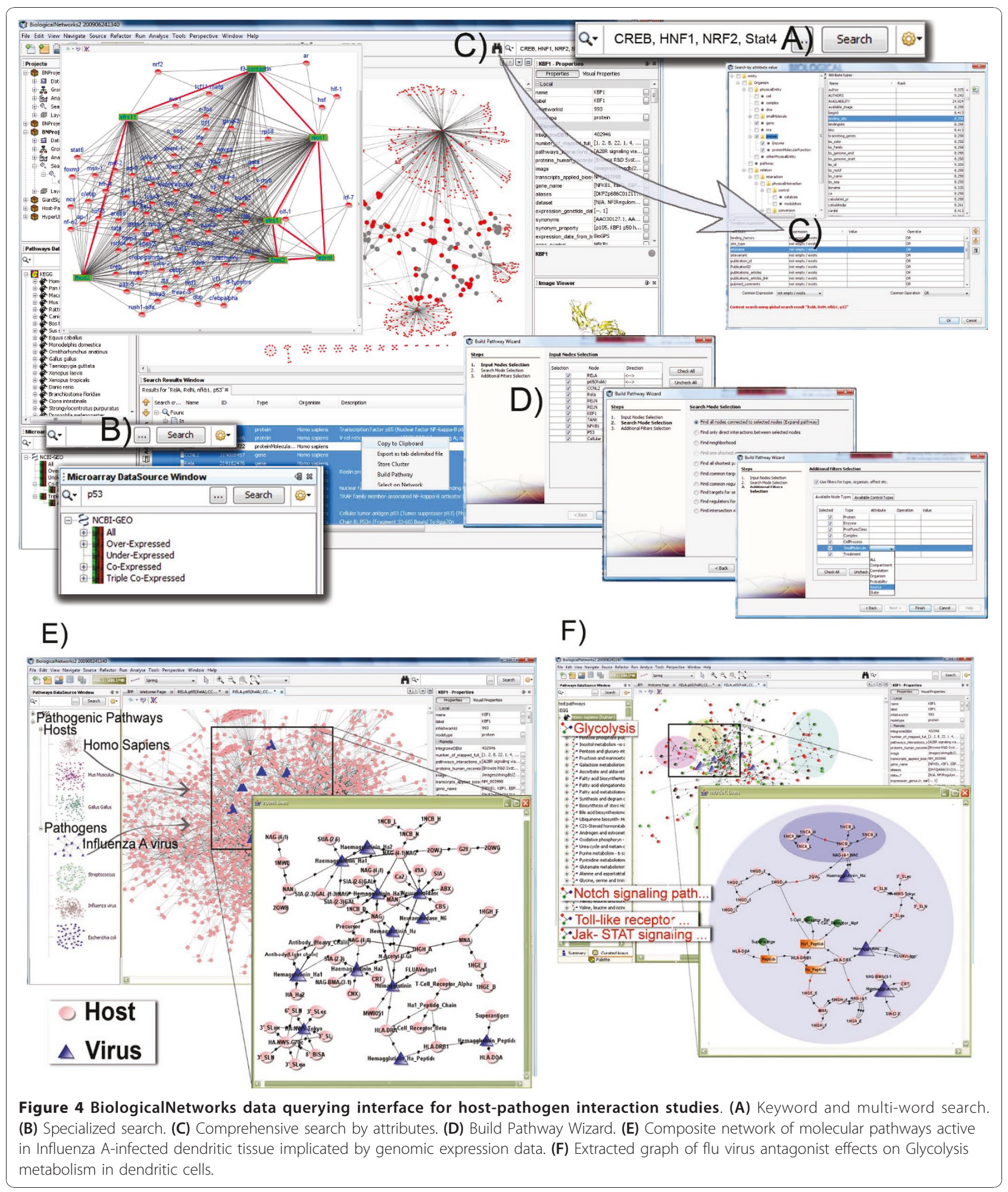

\section{Analyzing sub-networks}

In this section, we demonstrate the analysis of sub-networks and individual interactions in the interaction network.
First, we were interested in identifying sub-networks that were significantly induced or repressed relative to randomly selected sub-network [57]. Six such sub-networks were found; they overlapped and each consisted of 
about a hundred interactions. The pathways found in these sub-networks were consistent with a large-scale response of complex molecular pathways to the viral infection. Thus, genes involved in the interferon-response pathway were induced, owing to the immune response to viral infection. Nearly all genes involved in the Jak-STAT interferon-response signaling pathway and apoptosisrelated genes were activated; whereas the genes involved in the growth factors (IGF and connected pathways), cellcycle and translation-related pathways (CDKN and connected pathways) were repressed.

Second, six sub-networks were combined into a single fully-connected network (Figure 4F); that is, all interactions that did not belong any of the sub-network were excluded. In this network, we looked for the subnetworks that were significantly perturbed at early, middle or late stages of the influenza infection. As it was expected, "early" sub-networks contained pathways of the general immune response, whereas "middle" and "late" sub-networks - pathways specific for the infection. The genes involved in the pathways significant for early, middle and late stages are shown in Figure 4F and colored according to the stage. Further, we looked at the GeneOntology terms for the genes in the pathways that were perturbed at early, middle or late stages of the influenza infection. The following biological processes from GeneOntology were identified: immune response ( $p$-value $<1.0 \mathrm{e}-6)$, proteolysis and peptidolysis ( $p$-value $<1.0 e-5)$, lipid transport $(p$-value $<0.001)$, and complement activation $(p$-value $<0.01)$.

\section{Studying individual genes}

To study individual genes, we used the constructed in the previous section network of significantly induced/ repressed genes in the influenza infection (Figure 4F). For example, one can search for the genes that directly interact with the viral proteins and are known to be up- or down-regulated in human or mice (Query 3 in Additional File 1, Section S1.4). Thus, among the downregulated genes were the genes of the immune response (Toll-like receptors TLR1/TLR2 and interleukin), interferon-regulated genes (interferon-induced protein with tetratricopeptide repeats 2 (IFIT2) and vipirin), and the other genes involved in defense, inflammatory response and intracellular signaling pathways, including chemokine, apoptosis, MAPK, Notch, Jak-STAT, T-cell receptor, complement and coagulation cascades-pathways and genes are known to participate in the viral response and recruited by the virus for the entry $[17,58,59]$.

Further, we decided to focus on co-expressed genes. Such an analysis can give us host genes that can be potential targets for anti-viral drugs. We selected from the mouse network 45 co-expressed genes that were also differentially expressed in response to the influenza infection (Query 4 Additional File 1, Section S1.4). Since our database contains information on transcription factor binding sites, both experimental, predicted and conserved, we used that information to extend the network beyond the reported interactions. In the promoters of 45 co-expressed genes, we searched for binding sites that were conserved in the three species, Homo sapiens, Mus musculus and Rattus norvegicus. Such sites were found in 7 genes: SFRS11, SFRS1, FMNI2, LEPROT, NICN1, FHOD2, f3-contactin; if one is interested, the binding sites can be searched by sequence (Query 6 in Additional File 1, Section S1.4). Identified conserved binding sites were regulated by 73 transcription factors, including CREB, HNF1, NRF2, FOXP3, and factors from Pax, Gata and Stat families.

\section{Potential drug targets}

Which of the identified 7 co-expressed genes with phylogenetically conserved binding sites and their 73 potential transcription factors were not previously reported as associated with the influenza infection? The search (Query 7 in Additional File 1, Section S1.4) gave us 7 such genes/proteins: NFE2L2 (NRF2), FOXI1, SMAD6, HOXA3, SFRS11, GRAP, and AMPD1.

Based on available information, 5 genes, SMAD6, GRAP, HOXA3, NFE2L2, and AMPD1, might be suggested to be further investigated as drug targets in the influenza infection. Thus, SMAD6 is known to be involved in immune response (GO:0006955), signal transduction and transcriptional modulation of multiple signaling pathways, including BMP (GO:0030509) and TGF-beta receptor-signaling (GO:0030512). GRAP is involved in activation of the $\mathrm{T}$-cell antigen receptor (TCR) signal transduction pathway [60], Ras protein signal transduction (GO:0007265) and cell-cell signaling (GO:0007267). HOXA3 transcription factor may be required for the induction of pathogen-response genes in humans as it was shown that $S$. aureus infection induced a number of HOX genes that modulated the $\mathrm{NF}-\kappa \mathrm{B}$-dependent transcription and exerted this function redundantly [61].

AMPD, or AMP deaminase, is an enzyme that converts adenosine monophosphate (AMP) to inosine monophosphate (IMP), freeing an ammonia molecule in the process. Deficiency of this enzyme is a common cause of myopathy and rhabdomyolysis (the rapid breakdown of skeletal muscle). The influenza infections are known to be a cause of rhabdomyolysis, including seasonal [62] and recent H1N1 [63].

NFE2L2 gene codes the transcription factor NRF2 that is a known master regulator of the antioxidant response [64]. By inducing genes involved in combating oxidative stress that results in inflammation, neurological diseases, and renal disease, NRF2 protects body from a variety of 
oxidative stress-related complications. NRF2 activators have been studied as cancer [65] and diabetes drugs [66]. Our research shows that NRF2 might be considered a therapeutic candidate in the influenza infection as well.

\section{Adding phylogeography}

BiologicalNetwork allows phylogeographical analysis of a pathogen(s) strains/isolates. In particular, host-pathogen interaction networks for two or more pathogen genomes can be compared, using both phylogenetic and geographical data (Figure 5). Phylogeography seeks to explain the molecular evolution, genealogy and migration of species [54]. The strains in question are more likely to have similar pathogen-host interactions (and virulence properties) if they originated from the same geographical location and have relatively small phylogenetic distances [53,67]. Using phylogeographical approach, the closest phylogenetic neighbor for a new sequenced pathogen strain can be found, and its probability to be virulent can be estimated. The phylogeographical methods implemented in BiologicalNetworks are described in the section Methods and Demonstration page at http://flu.sdsc.edu/examples.jsp. Together with capability to integrate new phylogeographic data, these methods make BiologicalNetworks a unique among other integration systems. BiologicalNetworks allows visualizing and comparing host-pathogen networks in respect to the pathogens phylogenetic distance and geographic origin (Figure 5).

\section{Integration of user's data}

Any public or user's data in the table-format can be integrated into BiologicalNetworks automatically and studied together with other already integrated data. The user can do it at http://flu.sdsc.edu/integration.jsp (Figure 6A). We integrated host-pathogen interaction networks, pathways and all other data provided in the studies of Konig et. al. [17] and others [21-28]. Data from [17] are provided in 13 supplementary tables and contain: human cellular factors required for early-stage influenza virus replication, biochemical complexes that are required by different RNA viruses, host proteins confirmed to be required for wild-type influenza virus

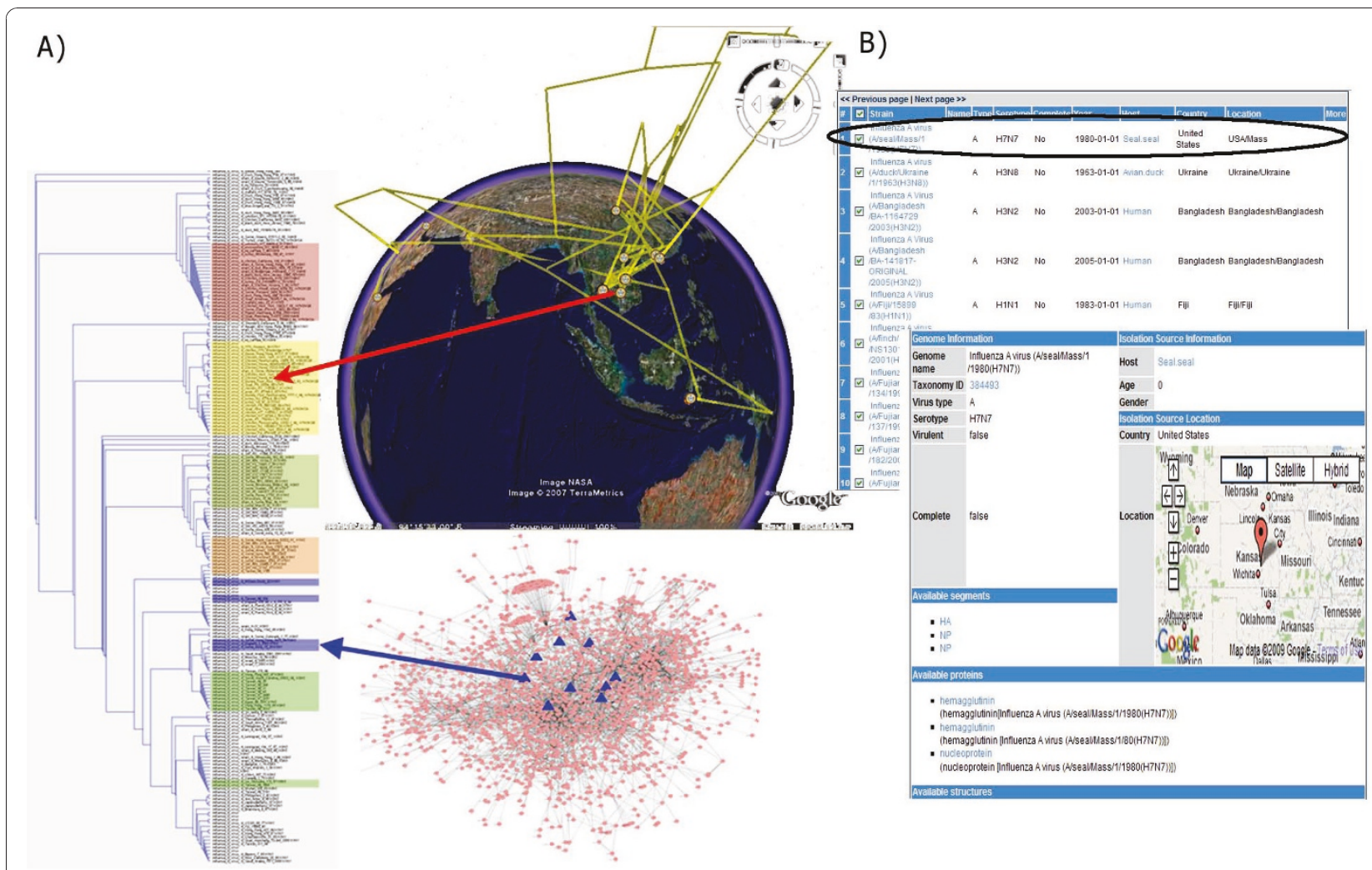

Figure $\mathbf{5}$ Visualization of phylogeographic data in Influenza virus study project. (A) Influenza virus clades are labeled by corresponding geographical region and visualized phylogenetically on GoogleEarth. Every spot on the globe corresponds to a particular strain of Influenza outbreak. Each leaf of the phylogenetic tree represents an influenza strain, and is located in the place where corresponding virus genome was found. This representation allows easily identify approximate location of the root node. The host-virus interaction network corresponds to one specific virus study. (B) Example of the genome detail page, with map showing location where a particular virus strain was isolated. 


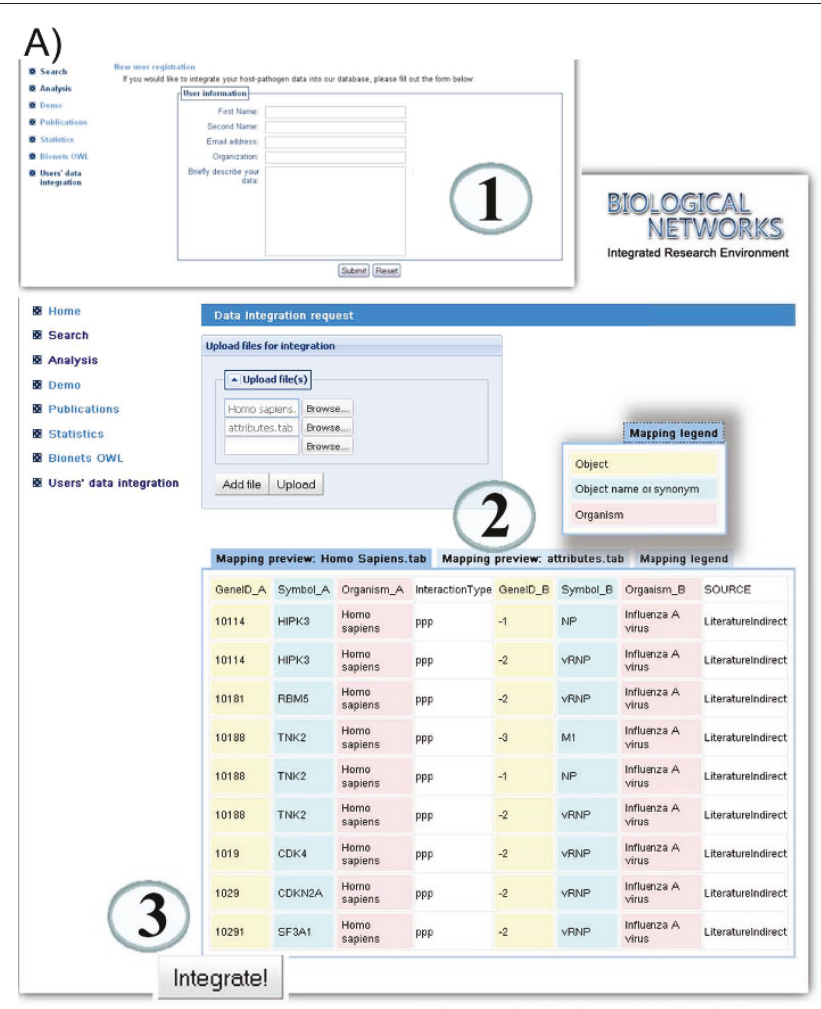

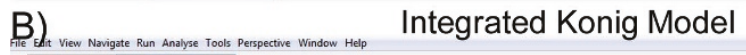

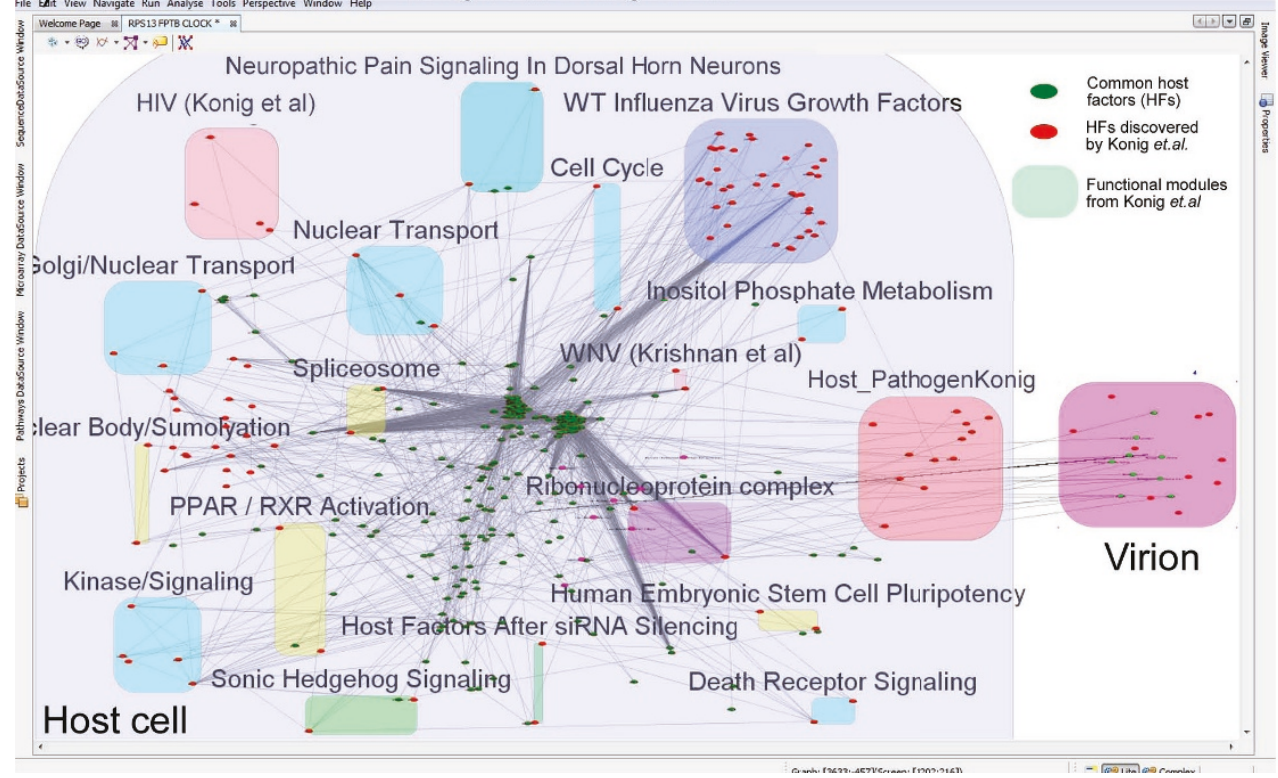

Figure 6 User's Data integration. (A) User's Data Integration page allows integrate host-pathogen data which is interesting for particular user but we didn't integrated. The integration procedure consists of three easy steps: 1) User registration, 2) Mapping of the data and 3) Integration of the data. We have integrated supplementary data provided in the studies of Konig et. al. [17] and others [21-28]. (B) Meta-network model of the Konig et. al. data integrated (in $\mathbf{A}$ )) into the IntegromeDB database and available to be analyzed in concert with wealth of other hostpathogen data available in BiologicalNetworks [40-42]. Host factors that were already present in our database and known to be related to viral response are colored green, whereas host factors newly discovered by Konig et. al. as being related to viral infection are colored red. Supplementary data from Konig et. al. integrated into our system are represented as colored meta-nodes (boxes): cyan- functional groups, yellow- molecular complexes, green - factors over-expressed after siRNA silencing, purple- biochemical complexes that are required by different RNA viruses, blue- host proteins confirmed to be required for wild-type influenza virus growth, pink -other virus experiments/publications (e.g. HIV, HCV, WNV, etc.) (see Supplementary data in Konig et. al.). 
growth, groups of genes over-expressed after siRNA silencing, other virus experiments/publications (e.g. HIV, HCV, WNV, etc.), etc. After automatic mapping and integrating those tables, we were able to visualize them as an integrated meta-network model in BiologicalNetworks. Now this meta-network model can be studied in relation to the data it was generated from-for example, different modules described in different data tables in relation to each other (Figure 6B, colored boxes)-and in relation to the other integrated data. For example, asking the system on what is known about the data imported from [17] (BuildPathwayWizard function "Find Meta Models" with specified parameters can be used for that), genes/proteins in the network can be seen in connection to the papers they were co-cited (Figure 6B) and/or mentioned together in supplementary materials. Similarly, the nodes in the network can be analyzed for co-expression, expression in particular tissues/cell types, and other functional information, such as functional modules, protein complexes and canonical pathways. The model is available for exploration either from BiologicalNetworks application as Demo Project or from the web site http://www.biologicalnetworks.org.

The ability to integrate Supplementary data for host-pathogen studies and to represent it in the digital integrated form is an extremely important feature for reproducible and integrated research. Several journals including Nature, PLoS, Cell are working towards establishing the reproducible research standards for their publications. In addition to asking the authors to represent their data in standard formats (e.g. MIAME for microarray data, SBML/SIF for network data, etc.), Cell journal, for example, now asks the authors to accompany their publications with graphical abstracts. Presenting the data in the form of a digital integrated model (e.g. BiologicalNetworks project file that can be opened on any computer) instead of the graphical picture would be much more useful for researchers and our future work will be towards that direction.

\section{Comparison with other systems}

We chose for comparison six resources: Cytoscape [29] and five resources developed specifically for studying host-pathogen interactions, PHI-base [12], PHIDIAS [13], PIG [14], IVDB (Influenza Virus Database) [15], and the NCBI Influenza Virus Database [16].

No two resources were similar by all 15 properties considered (Table 2). Most of the resources provide pathways and microarray data, however analysis and search of both types of data is provided only in Cytoscape and BiologicalNetworks. Phylogenetic analysis and sequence search are provided only in BiologicalNetworks and NCBI Influenza Virus Database. No resource except BiologicalNetworks is capable of analyzing regulatory regions, orthologous genes, 3D structural data, or dealing with phylogeographical data.

Due to the graph-based data integration model and the Semantic Web technologies implemented in BiologicalNetworks [42], it is scalable in respect to the number of integrated resources and therefore allows integration of user's data-this is the absolute merit of the proposed system for studying host-pathogen interactions.

Table 2 Comparison of BiologicalNetworks/HostPathogen database with public host-pathogen interaction resources

\begin{tabular}{llllllll}
\hline & $\begin{array}{l}\text { PHI- } \\
\text { base }\end{array}$ & PHIDIAS & $\begin{array}{l}\text { NCBI Influenza } \\
\mathbf{d b}\end{array}$ & PIG & IVDB & Cytoscape & $\begin{array}{l}\text { BiologicalNetworks/ } \\
\text { HostPathogenDB }\end{array}$ \\
\hline Scalability* & no & no & no & no & no & no & yes \\
\hline Data integration engine & no & no & no & no & no & no & yes \\
\hline $\begin{array}{l}\text { Interaction and Pathways data/ } \\
\text { analysis }\end{array}$ & yes & yes & no & yes & no & yes & yes \\
\hline Chemicals/Drug Discovery & no & no & no & no & no & yes & yes \\
\hline 3D structure & no & no & no & no & no & no & yes \\
\hline Sequence annotation/search & no/yes & yes/yes & yes/yes & no/ & no/no & no/no & yes/yes \\
\hline Phylogenetic analysis & no & no & yes & no & yes & no & yes \\
\hline Regulatory regions analysis & no & no & no & no & no & no & yes \\
\hline Orthology analysis & no & no & no & no & no & no & yes \\
\hline Phylogeoraphy & no & no & no & no & no & no & yes \\
\hline Microarray data/analysis & no/no & yes/no & yes/yes & yes/ & no/no & yes/yes & yes/yes \\
\hline $\begin{array}{l}\text { Web search/ } \\
\text { Research environment }\end{array}$ & yes/no & yes/no & yes/no & yes/ & yes/ & no/yes & yes/yes \\
\hline
\end{tabular}

*Scalability to the number of integrated data sources. 


\section{Conclusion}

BiologicalNetworks extensions for the host-pathogen studies enable diverse data in major human-disease systems to be subjected to efficient integrated analysis. The results show the utility of multi-scale data integration from large-scale human molecular-interaction, sequence and expression data to epidemiological and virulence data. The approach described allows information to be extracted that is not restricted to any one data type. Moreover, our analyses suggest how various host pathways act in response to viral infection, and serve as a large-scale window into the genomic response to Influenza and other respiratory infections. The pathways identified should provide insights into the mechanisms by which the host interacts with different pathogens, useful information about stage of disease, and selection of suitable targets for early diagnosis and treatments.

BiologicalNetworks has general purpose graph architecture and is data-type-neutral. Therefore, there is the prospect of further integration of data such as detailed clinical data that will enable clinical variables to be associated quantitatively with the activities of molecular pathways and processes. Capacity for integration gives our system a unique capability, the full potential of which will be realizable when a multitude of host-virus interaction data are available, so that similarities and differences between the interaction networks can be interrogated across the phylogenetic distance for more accurate prediction of the potential virulence of a newly isolated virus identified only by its sequence. We believe that the methods and tools we have implemented and described here will allow for the efficient dynamic integration and analysis of diverse data in other disease systems.

\section{Additional material}

Additional file 1: Methods. Detailed description of the methods and data types used in the BiologicalNetworks system for host-pathogen studies.

\section{Acknowledgements \\ This work was supported by the National Institute of Health grant R01GM084881 to MB, AG and AnR, R01GM085325 to JP, and Michael J. Connell Foundation grant to AnR and AG.}

\section{Author details}

'San Diego Supercomputer Center, University of California San Diego, 9500 Gilman Drive, La Jolla, CA, 92093, USA. ² Skaggs School of Pharmacy and Pharmaceutical Sciences, University of California San Diego, 9500 Gilman Drive, La Jolla, CA, 92093, USA. ${ }^{3}$ Keck Graduate Institute, 535 Watson Drive, Claremont, CA, 91711, USA.

\section{Authors' contributions}

SK, MS, AG and MB contributed to system concept. SK, MS, YD and MB implemented the system and performed major programming work. MB, JP and $A n R$ contributed to data analysis. This work was coordinated by AG, $A n R$, JP and MB. MB and JP wrote the manuscript. All authors read and approved the final manuscript.

Received: 23 November 2009 Accepted: 14 January 2011

Published: 14 January 2011

\section{References}

1. Ferguson NM, Cummings DA, Fraser C, Cajka JC, Cooley PC, Burke DS: Strategies for mitigating an influenza pandemic. Nature 2006, 442(27):448-452

2. Olsen B, Munster VJ, Wallensten A, Waldenstro J, Albert DM, Osterhaus E, Fouchier RA: Global Patterns of Influenza A Virus in Wild Birds. Science 2006, 312:384-388

3. May RM: Infectious Disease: Can We Avert a Lethal Flu Pandemic? Current Biology 2006, 15(22):922-924.

4. Neumann G, Noda T, Kawaoka Y: Emergence and pandemic potential of swine-origin H1N1 influenza virus. Nature 2009, 459:931-939.

5. Morens DM, Taubenberger JK: Understanding influenza backward. JAMA 2009, 302:679-680

6. Munster VJ, de Wit E, Brand JM, Herfst S, Schrauwen EJ, Bestebroer TM, Vijver D, Boucher CA, Koopmans M, Rimmelzwaan GF, Kuiken T, Osterhaus AD, Fouchier RA: Pathogenesis and transmission of swineorigin $2009 \mathrm{~A}(\mathrm{H} 1 \mathrm{~N} 1)$ influenza virus in ferrets. Science 2009, 325:481-483.

7. Kaufman MA, Duke GJ, McGain F, French C, Aboltins C, Lane G, Gutteridge GA: Life-threatening respiratory failure from H1N1 influenza 09 (human swine influenza). Med J Aust 2009, 191:154-156.

8. Centers for Disease Control and Prevention (CDC): Intensive-care patients with severe novel influenza A (H1N1) virus infection - Michigan, June 2009. MMWR Morb Mortal Wkly Rep 2009, 58:749-752.

9. Kelly H, Grant K: Interim analysis of pandemic influenza (H1N1) 2009 in Australia: surveillance trends, age of infection and effectiveness of seasonal vaccination. Euro Surveill 2009, 14:19288.

10. Itoh $Y$, Shinya K, Kiso M, Watanabe T, Sakoda $Y$, Hatta M, Muramoto $Y$, Tamura D, Sakai-Tagawa Y, Noda T, Sakabe S, Imai M, Hatta Y, Watanabe S, Li C, Yamada S, Fujii K, Murakami S, Imai H, Kakugawa S, Ito M, Takano R, Iwatsuki-Horimoto K, Shimojima M, Horimoto T, Goto H, Takahashi K, Makino A, Ishigaki $H$, Nakayama $M$, et al: In vitro and in vivo characterization of new swine-origin $\mathrm{H} 1 \mathrm{~N} 1$ influenza viruses. Nature 2009, 460:1021-1025.

11. Maines TR, Jayaraman A, Belser JA, Wadford DA, Pappas C, Zeng H, Gustin KM, Pearce MB, Viswanathan K, Shriver ZH, Raman R, Cox NJ, Sasisekharan R, Katz JM, Tumpey TM: Transmission and pathogenesis of swine-origin $2009 \mathrm{~A}(\mathrm{H} 1 \mathrm{~N} 1)$ influenza viruses in ferrets and mice. Science 2009, 325:484-487.

12. Winnenburg $R$, Urban $M$, Beacham A, Baldwin TK, Holland S, Lindeberg M, Hansen H, Rawlings C, Hammond-Kosack KE, Kohler J: PHI-base update: additions to the pathogen host interaction database. Nucleic Acids Res 2008, 36:D572-576.

13. Zuoshuang Xiang, Yuying Tian, Yongqun He: PHIDIAS: a pathogen-host interaction data integration and analysis system. Genome Biology 2007, 8: R150.

14. Driscoll T, Dyer MD, Murali TM, Sobral BW: PIG-the pathogen interaction gateway. Nucleic Acids Res 2009, 37:D647-D650.

15. Chang S, Zhang J, Liao X, Zhu X, Wang D, Zhu J, Feng T, Zhu B, Gao GF, Wang J, Yang H, Yu J, Wang J: Influenza Virus Database (IVDB): an integrated information resource and analysis platform for influenza virus research. Nucleic Acids Res 2007, 35:D376-D380.

16. Bao Y, Bolotov P, Dernovoy D, Kiryutin B, Zaslavsky L, Tatusova T, Ostell J, Lipman D: The Influenza Virus Resource at the National Center for Biotechnology Information. J. Virol 2008, 82(2):596-601.

17. Konig R, Stertz S, Zhou Y, Inoue A, Hoffmann HH, Bhattacharyay S, Alamares J, Tscherne DM, Ortigoza MB, Liang Y, Gao Q, Andrews SE, Bandyopadhyay S, De Jesus P, Tu B, Pache L, Shih C, Orth A, Bonamy G, Miraglia L, Ideker T, Garcia-Sastre A, Young JAT, Palese P, Shaw ML, Chanda SK: Human Host Factors Required for Influenza Virus Replication. Nature 2009, 463(7282):813-7.

18. Gersten M, Alirezaei M, Marcondes MC, Flynn C, Ravasi T, Ideker T, Fox HS An integrated systems analysis implicates EGR1 downregulation in SIVE-induced neural dysfunction. Journal of Neuroscience 2009, 29(40):12467-76. 
19. Fossum E, Friedel CC, Rajagopala SV, Titz R, Baiker A, Schmidt T, Kraus T, Stellberger T, Rutenberg C, Suthram S, Bandyopadhyay S, Rose D, von Brunn A, Uhlmann M, Zeretzke C, Dong YA, Boulet H, Koegl M, Bailer SM, Koszinowski U, Ideker T, Uetz P, Zimmer R, Haas J: Evolutionarily conserved herpesviral protein interaction networks. PLoS Pathogens 2009, 5(9): e1000570.

20. Bushman FD, Malani N, Fernandes J, D'Orso I, Cagney G, Diamond TL, Zhou H, Hazuda DJ, Espeseth AS, Konig R, Bandyopadhyay S, Ideker T, Goff SP, Krogan NJ, Frankel AD, Young JA, Chanda SK: Host cell factors in HIV replication: meta-analysis of genome-wide studies. PLOS Pathog 2009, 5(5):e1000437.

21. Good BM, Wilkinson MD: The Life Sciences Semantic Web is full of creeps! Brief Bioinform 2006, 7(3):275-286.

22. Brass AL, Dykxhoorn DM, Benita Y, Yan N, Engelman A, Xavier RJ, Lieberman J, Elledge SJ: Identification of host proteins required for HIV infection through a functional genomic screen. Science 2008, 319:921-926, 2008.

23. König $R$, Zhou $Y$, Elleder $D$, Diamond $T L$, Bonamy GMC, Irelan JT, Chiang $C$, Tu BP, De Jesus PD, Lilley CE, Seidel S, Opaluch AM, Caldwell JS, Weitzman MD, Kuhen KL, Bandyopadhyay S, Ideker T, Orth AP, Miraglia LJ, Bushman FD, Young JA, Chanda SK: Global analysis of host-pathogen interactions that regulate early-stage HIV-1 replication. Cell 2008, 135:49-60.

24. Zhou H, Xu M, Huang Q, Gates AT, Zhang XD, Castle JC, Stec E, Ferrer M, Strulovici B, Hazuda DJ, Espesethet AS: Genome-scale RNAi screen for host factors required for HIV replication. Cell Host Microbe 2008, 4:495-504.

25. Krishnan MN, Ng A, Sukumaran B, Gilfoy FD, Uchil PD, Sultana H, Brass AL, Adametz R, Tsui M, Qian F, Montgomery RR, Lev S, Mason PW, Koski RA, Elledge SJ, Xavier RJ, Agaisse H, Fikrig E: RNA interference screen for human genes associated with West Nile virus infection. Nature 2008, 455:242-245.

26. Tai AW, Benita Y, Peng LF, Kim SS, Sakamoto N, Xavier RJ, Chung RT: A functional genomic screen identifies cellular cofactors of hepatitis $C$ virus replication. Cell Host Microbe 2009, 5:298-307.

27. Sessions OM, Barrows NJ, Souza-Neto JA, Robinson TJ, Hershey CL, Rodgers MA, Ramirez JL, Dimopoulos G, Yang PL, Pearson JL, GarciaBlanco MA: Discovery of insect and human dengue virus host factors. Nature 2009, 458:1047-1050.

28. Hao L, Sakurai A, Watanabe T, Sorensen E, Nidom CA, Newton MA, Ahlquist $P$, Kawaoka Y: Drosophila RNAi screen identifies host genes important for influenza virus replication. Nature 2008, 454:890-893.

29. Shannon P, Markiel A, Ozier O, Baliga NS, Wang JT, Ramage D, Amin N, Schwikowski B, Ideker T: Cytoscape: a software environment for integrated models of biomolecular interaction networks. Genome Res 2003, 13:2498-504

30. Salomonis N, Hanspers K, Zambon AC, Vranizan K, Lawlor SC, Dahlquist KD, Doniger SW, Stuart J, Conklin BR, Pico AR: GenMAPP 2: new features and resources for pathway analysis. BMC Bioinformatics 2007, 8:217.

31. Sayers EW, Barrett T, Benson DA, Bryant SH, Canese K, Chetvernin V, Church DM, DiCuccio M, Edgar R, Federhen S, Feolo M, Geer LY, Helmberg W, Kapustin Y, Landsman D, Lipman DJ, Madden TL, Maglott DR, Miller V, Mizrachi I, Ostell J, Pruitt KD, Schuler GD, Sequeira E, Sherry ST, Shumway M, Sirotkin K, Souvorov A, Starchenko G, Tatusova TA, Wagner L, Yaschenko E, Ye J: Database resources of the National Center for Biotechnology Information. Nucleic Acids Res 2009, 37:D5-15.

32. Hubbard TJ, et al: Ensembl 2009. Nucleic Acids Res 2009, 37:D690-697.

33. Haider S, Ballester B, Smedley D, Zhang J, Rice P, Kasprzyk A: BioMart Central Portal-unified access to biological data. Nucleic Acids Res 2009, 37:W23-27.

34. von Mering $C$, Jensen $L$, Snel B, Hooper SD, Krupp, Foglierini M, Jouffre $N$, Huynen MA, Bork P: STRING: known and predicted protein-protein associations, integrated and transferred across organisms. Nucleic Acids Res 2007, 33:D433-7.

35. Safran M, Chalifa-Caspi V, Shmueli O, Olender T, Lapidot M, Rosen N, Shmoish M, Peter Y, Glusman G, Feldmesser E, Adato A, Peter I, Khen M, Atarot T, Groner Y, Lancet D: Human Gene-Centric Databases at the Weizmann Institute of Science: GeneCards, UDB, CroW 21 and HORDE. Nucleic Acids Res 2003, 1:142-146.

36. Hernandez-Boussard T, Woon M, Klein TE, Altman RB: Integrating largescale genotype and phenotype data. OMICS 2006, 10(4):545-54.
37. Kohler J, Baumbach J, Taubert J, Specht M, Skusa A, Ruegg A, Rawlings C, Verrier P, Philippi S: Graph-based analysis and visualization of experimental results with ONDEX. Bioinformatics 2009, 22(11):1383-1390.

38. Birkland A, Yona G: BIOZON: a system for unification, management and analysis of heterogeneous biological data. BMC Bioinformatics 2006, 7:70.

39. Kuntzer J, Backes C, Blum T, Gerasch A, Kaufmann M, Kohlbacher O, Lenhof HP: BNDB - the Biochemical Network Database. BMC Bioinformatics 2007, 8:367.

40. Baitaluk M, Qian X, Godbole S, Raval A, Ray A, Gupta A: PathSys: Integrating molecular interaction graphs for systems biology. BMC Bioinformatics 2006, 7:55.

41. Baitaluk M, Sedova M, Ray A, Gupta A: BiologicalNetworks: visualization and analysis tool for systems biology. Nucleic Acids Res 2006, 34 W466-W471.

42. Baitaluk M, Ponomarenko J: Semantic integration of data on transcriptional regulation. Bioinformatics 2010

43. The Open Biomedical Ontologies. [http://www.obofoundry.org/index.cgi? show=mappings].

44. Krishnamurthy N, Brown D, Kirshner D, Sjolander K: PhyloFacts: an online structural phylogenomic encyclopedia for protein functional and structural classification. Genome Biology 2006, 7:R83.

45. Gusfield D: Algorithms on Strings, Trees, and Sequences. Cambridge University Press; 1997.

46. Farach-Colton M: Optimal suffix tree construction with large alphabets. 39th Annual Symposium on Foundations of Computer Science 1997.

47. Farach-Colton M, Ferragina P, Muthukrishnan S: Overcoming the memory bottleneck in suffix tree construction. 39th Annual Symp. on Foundations of Computer Science 1998.

48. Farach-Colton $M$, Ferragina $P$, Muthukrishnan S: On the sorting-complexity of suffix tree construction. Journal of the ACM 2000, 47(6):987-1011.

49. Giegerich R, Kurtz S, Stoye J: Efficient implementation of lazy suffix trees. Software Practice \& Experience 2003, 33(11):1035-1049.

50. Ukkonen E, Kärkkäinen J: Sparse suffix trees. 2nd Annual Int'l Conference on Computing and Combinatorics 1996.

51. Phoophakdee B, Zaki MJ: TRELLIS+: an effective approach for indexing genome-scale sequences using suffix trees. Pac. Symp. Biocomput 2008, 90-101.

52. Seshadri R, Kravitz SA, Smarr L, Gilna P, Frazier M: CAMERA: A Community Resource for Metagenomics. PLoS Biol 2007, 5(3):e75.

53. Duan L, et al: Characterization of Low-Pathogenic H5 Subtype Influenza Viruses from Eurasia: Implications for the Origin of Highly Pathogenic H5N1 Viruses. Journal of Virology 2007, 81(14):7529-7539.

54. Wallace RG, HoDac HM, Lathrop RH, Fitch WM: A statistical phylogeography of influenza A H5N1. Proc Natl Acad Sci USA 2007, 104:4473-4478

55. Edgar RC: MUSCLE: multiple sequence alignment with high accuracy and high throughput. Nucleic Acids Res 2004, 32(5):1792-1797.

56. Chang LW, Nagarajan R, Magee JA, Milbrandt J, Stormo GD: A systematic model to predict transcriptional regulatory mechanisms based on overrepresentation of transcription factor binding profiles. Genome Res 2006, 16:405-413.

57. Ideker T, Ozier O, Schwikowski B, Siegel A: Discovering regulatory and signalling circuits in molecular interaction networks. Bioinformatics 2002, 18:233-240.

58. Malmgaard L: Induction and regulation of IFNs during viral infections. J Interferon Cytokine Res 2004, 24(8):439-54.

59. Samuel CE: Antiviral actions of interferons. Clin Microbiol Rev 2001, 14(4):778-809.

60. Zhang W, Sloan-Lancaster J, Kitchen J, Trible RP, Samelson LE: LAT: the ZAP-70 tyrosine kinase substrate that links $\mathrm{T}$ cell receptor to cellular activation. Cell 1998, 92(1):83-92.

61. Irazoqui JE, Ng A, Xavier RJ, Ausubel FM: Role for beta-catenin and HOX transcription factors in Caenorhabditis elegans and mammalian host epithelial-pathogen interactions. Proc Natl Acad Sci 2008, 105(45):17469-74

62. Buss BF, Shinde VM, Safranek TJ, Uyeki TM: Pediatric influenza-associated myositis - Nebraska, 2001-2007. Influenza Other Respi Viruses. 2009, 3(6):277-85

63. Parikh M, Dolson G, Ramanathan $V$, Sangsiraprapha W: Novel H1N1associated rhabdomyolysis leading to acute renal failure. Clin Microbiol Infect 2010, 16(4):330-2. 
64. Nguyen T, Nioi P, Pickett CB: The Nrf2-antioxidant response element signaling pathway and its activation by oxidative stress. J Biol Chem 2009, 284(20):13291-5.

65. Zhang Y, Gordon GB: A strategy for cancer prevention: stimulation of the Nrf2-ARE signaling pathway. Mol Cancer Ther 2004, 3(7):885-93.

66. Brownlee M: Biochemistry and molecular cell biology of diabetic complications. Nature 2001, 13:813-20, 414(6865).

67. Gertz J, Elfond G, Shustrova A, Weisinger M, Pellegrini M, Cokus S,

Rothschild B: Inferring protein interactions from phylogenetic distance matrices. Bioinformatics 2003, 19:2039-45.

doi:10.1186/1752-0509-5-7

Cite this article as: Kozhenkov et al:: BiologicalNetworks - tools enabling the integration of multi-scale data for the host-pathogen studies. BMC

Systems Biology 2011 5:7.

Submit your next manuscript to BioMed Central and take full advantage of:

- Convenient online submission

- Thorough peer review

- No space constraints or color figure charges

- Immediate publication on acceptance

- Inclusion in PubMed, CAS, Scopus and Google Scholar

- Research which is freely available for redistribution

Submit your manuscript at www.biomedcentral.com/submit 